astrocytes directly onto the rat's brains, or when they blocked the expression of lactate transporters in the hippocampus, the rats were unable to recall which part of the cage to avoid.

Cell 144, 810-823 (2011)

\section{ASTRONOMY}

\section{New-looking old galaxies}

Using a combination of space and ground-based telescopes, astronomers have spotted the most distant, and hence earliest, cluster of galaxies ever seen. The cluster dates to a time when the Universe was just a quarter of its current age of 13.7 billion years, yet, surprisingly, it looks more like nearby, modern galaxy clusters than the starforming proto-clusters found so far at the same epoch.

Raphael Gobat of the Laboratory for Astrophysics Instrumentation and Modelling in Gif-sur-Yvette, France, and his colleagues say that more observations should show whether the cluster is a fluke, or whether theories of cluster formation need to be revised.

Astron. Astrophys. 526, A133

(2011)

\section{MICROBIOLOGY}

\section{Stopping the cellular pump}

The bacterium responsible for tuberculosis, Mycobacterium tuberculosis, is notorious for its tolerance to antibiotics. It enters a dormant phase, which, it was assumed, makes it less sensitive to drugs that target dividing bacteria. But it seems that even replicating mycobacteria can fend off antibiotics.

Within days of infecting zebrafish larvae with Mycobacterium marinum, Lalita Ramakrishnan at the University of Washington in Seattle and her colleagues found dividing drug-tolerant bacteria inside macrophages, a type of immune cell. In this environment, the mycobacteria synthesized molecular 'pumps' that expel drugs from the bacterial cell and promote its replication.

When the team treated cultured human macrophages infected with $M$. tuberculosis with verapamil, a pump inhibitor, drug tolerance decreased, suggesting that verapamil-like drugs might be able to shorten the current sixmonth-minimum treatment regimen for tuberculosis. Cell doi:10.1016/j.cell. 2011.02.022 (2011)

\section{MATERIALS}

\section{Fine-tuning optical fibres}

Light-transmitting fibres in fibre-optic devices are mostly made of glasses such as silica. The fabrication of crystalline compound semiconductor fibres made of zinc selenide (ZnSe), which have a wider range of desirable properties than previous fibres, opens up the possibility of developing fibre-based lasers and amplifiers for photonic applications.

John Badding at Pennsylvania State University in University Park and his collaborators deposited $\mathrm{Zn}$ and Se inside a hollow silica fibre (pictured) using a technique for high-pressure chemical vapour deposition. The micrometresized fibres had low loss of light transmission in the mid-infrared region. Their optical characteristics can be tuned by changing the composition of the material, the authors say. Adv. Mater. doi:10.1002/ adma.201003214 (2011)

\section{NEUROSCIENCE}

\section{Brain fluid spurs stem cells}

The cerebrospinal fluid (CSF) that bathes the brain and spinal cord seems to be more than just a cushion against shock. Key molecules in the CSF signal

COMMUNITY CHOICE

The most viewed papers in science

\title{
New proteins from neighbours
}

\section{HIGHLY READ \\ on www.plosgenetics. org the weeks of 21 and 28 February}

Bacteria are highly adaptive, constantly creating new proteins. Most of the genes for these proteins have been acquired from other organisms, and did not arise from the duplication and mutation of the microbes' own genes as previously thought.

Todd Treangen and Eduardo Rocha at the Pasteur Institute in Paris determined the source of more than 3,000 families of proteins in 110 bacteria belonging to 8 different groups. Depending on the group, between $88 \%$ and $98 \%$ of the new genes in the species studied came from other bacteria through a process known as horizontal gene transfer.

New proteins resulting from gene transfer evolve faster and persist longer than those encoded by duplicated genes, the authors show.

PLoS Genet. 7, e1001284 (2011) neural stem cells to proliferate, and may be involved in a type of brain cancer.

Christopher Walsh at the Children's Hospital Boston in Massachusetts and his colleagues found that membrane proteins on the surface of neural stem cells facing the CSF bind to proteins, including insulinlike growth factor 2 (Igf2), in the fluid. Blocking this binding in mice resulted in animals with small brains. Patients with a type of brain tumour were found to have higher than normal levels of Igf 2 in their CSF. Neuron 69, 893-905 (2011) IMMUNOLOGY

\section{Feedback loop in lupus}

The second most common human autoimmune disease, systemic lupus erythematosus (SLE), is marked by overproduction of antibodies against the body's nucleic acids. Two studies show that white blood cells called neutrophils from patients with lupus release a network of DNA-rich fibres called neutrophil extracellular traps (NETs) that may sustain the disease.

Virginia Pascual at the Baylor Institute for Immunology Research in Dallas, Texas, and her colleagues found that neutrophils from children with SLE die in vivo at higher rates than those from healthy individuals. When exposed to antibodies found in the blood of patients with SLE, dying neutrophils produce NETs that activate cells called plasmacytoid dendritic cells. These produce high levels of interferon- $\alpha$ - which triggers immune responses, thus priming more neutrophils to release more NETs.

Michel Gilliet at the University of Texas, Houston, and his colleagues showed that people with lupus also generate antibodies against antimicrobial peptides carried by NETs, suggesting that the NETs trigger additional autoantibody production. Sci. Trans. Med. 3, 73ra19; 73ra20 (2011)

\section{$\rightarrow$ NATURE.COM}

For the latest research published by Naturevisit:

www.nature,com/latestresearch 